\title{
MOBILE TRAFFIC ALERT AND TOURIST ROUTE GUIDANCE SYSTEM DESIGN USING GEOSPATIAL DATA
}

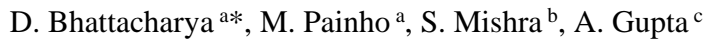 \\ a Nova Information Management School, Universidade Nova de Lisboa, Lisbon, Portugal - (dbhattacharya, painho)@ novaims.unl.pt \\ ${ }^{b}$ Dept. of Electronics and Communication Engg., Dr.A.P.J.Abdul Kalam Technical University, G.C.E.T., India - \\ sumitmishra209@gmail.com \\ ${ }^{c}$ Department of Civil Engineering, Indian Institute of Technology (Banaras Hindu University), Varanasi, India - \\ ankit.civ@iitbhu.ac.in
}

Commission IV, WG IV/10

KEY WORDS: Traffic alert, Mobile communication, Intelligent traffic information system, Geo-information technology, Traffic management, Short message service, geo-spatial information dissemination, geo-reference.

\begin{abstract}
:
The present study describes an integrated system for traffic data collection and alert warning. Geographical information based decision making related to traffic destinations and routes is proposed through the design. The system includes a geospatial database having profile relating to a user of a mobile device. The processing and understanding of scanned maps, other digital data input leads to route guidance. The system includes a server configured to receive traffic information relating to a route and location information relating to the mobile device. Server is configured to send a traffic alert to the mobile device when the traffic information and the location information indicate that the mobile device is traveling toward traffic congestion. Proposed system has geospatial and mobile data sets pertaining to Bangalore city in India. It is envisaged to be helpful for touristic purposes as a route guidance and alert relaying information system to tourists for proximity to sites worth seeing in a city they have entered into. The system is modular in architecture and the novelty lies in integration of different modules carrying different technologies for a complete traffic information system. Generic information processing and delivery system has been tested to be functional and speedy under test geospatial domains. In a restricted prototype model with geo-referenced route data required information has been delivered correctly over sustained trials to designated cell numbers, with average time frame of 27.5 seconds, maximum 50 and minimum 5 seconds. Traffic geo-data set trials testing is underway.
\end{abstract}

\section{INTRODUCTION AND BACKGROUND}

Increased traffic congestion is an ever-increasing problem in major urban areas like Bangalore. Traffic congestion has an adverse effect on the environment and adds stress to peoples' daily lives. It is important that accurate traffic information reaches those on road in real time. Accurate traffic information allows not only traffic engineers to pin point problem areas, but also provides drivers with near real time information to avoid problems. And with mobile phone being a communication device of choice for 9 million of the 9.5 million Bangalore population, it could be used very effectively in gathering information of traffic movements around junctions and notify traffic abnormalities back to the mobiles via messages which is the idea behind developing this system. With text capable mobiles being ubiquitous rather than smart-phones, so it is wise to issue text traffic alerts for city wide coverage (Sun and Liu, 2015).

Several traffic monitoring systems and methods currently exist. Some of the current traffic monitoring systems are crude stand alone devices that merely count the number of cars that pass over a sensor (Yuan et al., 2014). In order to gather any information from these devices a person must go out to the device and read the counter at the location, or take the device from the location where the counter can be read (Semertzidis et al., 2010). Such devices do not provide real time information. Moreover, such devices do not provide any sort of error detection to alert traffic engineers if the device is malfunctioning (Jog et al., 2011). Other traffic monitoring systems are configured to provide real time or near real time information. Such systems typically comprise remote traffic monitoring units that communicate in some way with a central station. In some of the prior art systems the remote units are hardwired, such as through telephone lines, to a central station. As such, the remote units of these systems are typically permanently fixed to a location and are not easily moved. These devices are typically "dumb" monitoring devices with no localized processing capability. Moreover, these devices typically do not monitor roadway conditions and do not have the capability to record traffic for specified pre-determined periods (Piao et al., 2012). Some prior art systems exist that utilize cellular or radio transmission to communicate from the remote monitoring devices to a central station. With these cellular systems, the voice channels are typically used to transmit monitored data. Such systems use up valuable spectrum space on the voice channels and depending on the size of the system may require additional capacity to be added to the cellular system (Manolis et al., 2016). The systems that utilize radio transmission require that a radio network be built to accommodate the system. As such, these methods of transmission are expensive to implement. Hence we suggest a better more popular technology based solution that could be readily adopted without much investment.

\section{PRIOR ART AND SYSTEM INNOVATIVENESS}

Traffic alert system effectiveness is governed by operating and maintenance overheads (Ma et al., 2015). Traffic alert systems have reportedly required expert operators and sensitive handling (Ajitha et al., 2013). Present traffic systems

\footnotetext{
* Corresponding author
} 
are infrastructure intensive and hard to deploy, maintain and upgrade (Barone et al., 2014). Experienced personnel are required to administer such systems, hence preventing wide usage. In the hour of need comprehensible and intelligible systems serve the masses better.

The proposed system should be able to decipher traffic alert information, able to make decisions fast, and communicate. It has to have a degree of automation for realtime action. The yardstick for performance of an traffic alert system is the number of people it reaches in as minimum time as possible (Korhonen et al., 2012). The efficiency of a traffic alert system can be enhanced through the use of extensive mediums of telecommunication and real-time range over a mass medium to as many people (Manasseh and Sengupta, 2010).

The system should not be limited in extent. It should be ubiquitous. The stipulation is for in time individual notification in the hour of need. Prevalent communication technologies use may be researched to increase the effectiveness of the system. Engineering aspects have to deal with effect of local telecom disturbances hampering the traffic alert system also, hence a system which can tolerate local bottlenecks to a large extent and keep functioning, has to be researched.

Existing early traffic alert systems are based on intensive dedicated infrastructure but not SMS (Zhao et al., 2012). The existing systems show drawbacks such as working for one or two site-specific traffic situation alerts. And most of these are authoritative traffic alert systems and cannot disseminate automated traffic alert to the affected people (Zhao et al., 2012).

A value added traffic alert system would be one capable of disseminating adequate and timely traffic and situational information to the public (Cervantes et al., 2016). It should be capable of leveraging geographical information system (GIS) compatible datasets as well as be able to handle location through latitude, longitude data from global positioning systems (GPS). An automated and generalized architecture with minimum setup requirement is needed for a system to be practically implemented (Zarif et al., 2003). Further to promote popular usage and quick and easy use, a traffic alert system using prevalent technology has to be designed (Pascale et al., 2012). To reach affected people in time it makes sense to develop with available infrastructure, mechanisms and set-ups. More so by way of short message service (SMS) which has been widely documented to be effective for alerts (Sisiopiku and Elliott, 2005). The burgeoning number of mobile subscriptions around the world facilitates reaching more than eighty-five percent of population on an average in any given zone. Moreover mobile communication infrastructure is ubiquitous and bandwidth manipulation has research scope for continuous information delivery (Bhattacharya et al., 2011). Finally, a traffic alert communication system which can be easily integrated to any traffic situation evaluation system will really help to augment current capability (Bhattacharya et al., 2013).

The concept of an automated alert system addresses many complex issues related to traffic alerts (Bhattacharya et al., 2014). Such a system can tie together traffic situation data and alert mechanism based on inbuilt knowledge base, be centrally coordinated and run multiple tasks simultaneously and repeatedly, thereby emulating multiple expert groups (Sisiopiku and Elliott, 2005). The content of traffic alert messages may originate with different monitoring devices and groups, but it is important that messages to the public are consistent with one another and their dissemination coordinated to achieve the best effect (Manolis et al., 2016). In large, rapidly emerging traffic events, coordination between data collection, supporting, and managing agencies requires a high level of overhead and maintaining this effort in the context of an emergency is a tall order (Wang et al., 2016). Hence to address these challenging issues the development of an automated traffic alert system is needed.

The need for a reliable, coordinated approach to disseminating information originating from diverse traffic situations and scenarios has led to ideas for combined traffic scene evaluation and alert information (Korhonen et al., 2012). Hence it is necessary to maintain multitasking and centralized control, which could be well imparted through an expertly designed traffic alert system. Once this is achieved, multiple purposes can be served like touristic places information dissemination, public services information etc.

There have been separate systems for traffic alert as well as for assessment of traffic situation but their integration has been problematic. This leaves incompleteness as far as the information of end-user is concerned. A traffic alert system with the ability to receive and understand information from external source and forward it intelligently as advisory, could be a solution to the problem. The information could be the output of another system viz. traffic and location details assessment system. This requires development of traffic alert communication system having functionality to associate with external information as used for imminent alerts (Bhattacharya et al., 2011, 2013, 2014).

Thus, the proposed traffic alert system consists of an input subsystem, a processing subsystem, and a traffic alert subsystem shown in Figure 1. The mutual interactions among the different subsystems, through predefined rules, make the system feasible and workable. Described the components (Figure 1) of a generalized traffic alert system, their broad functionalities and an overview of the interactive monitoring and responses. The structure is valid generally and can be improved upon by focusing on the respective subsystems. The challenges associated with the development of a traffic alert communication system are:

- Designing of popular usage based traffic alert communication system through integration of prevalent technologies;

- Integration of traffic evaluation system with the traffic alert communication system; and

- Development of methodology for automated working of the system.

\section{METHODOLOGY}

The working of any traffic communication system requires four basic functions: accepting / receiving, processing, storing and transferring of data (Figure 1). These broad functionalities could be provided by the combined working of the information and communications technologies along with the framework of geo-spatial technology.

The integration of two diverse functionalities of traffic evaluation and traffic alert requires the introduction of a common platform. One possible solution could be a system capable of inculcating information regarding possibility of traffic and use available communication infrastructure to notify the same to the public. The communication of traffic alert to the end-users may be realized by communicating over the standing cellular network.

One of the good practices of system design is to keep it modular and compartmentalize the tasks according to the modules. There has to be transmission of data between modules in a system for which well-defined prior mutual agreements are 
required among the modules. Hence, the gaps in knowledge towards research scope are:

1. The integration of alert proclamation and warning communication in a standalone system yet to be achieved.

2. The existing warning systems lack easy deploy ability, operability and replicability. It is seen that such researches stay confined to the scientific community only.

\section{The system needs to reach individuals.}

4. A modular structure of addressing the problem of alert prediction and warning is lacking. It is imperative that there needs to be comprehensive autonomy to each functional part of the system which is missing in current literature.

5. A traffic alert system hybridizing the concepts of the widespread technologies of communication such as web-technology and mobile technology is a worthwhile area to be researched.

Thus the components of research that need to be addressed for the gaps in knowledge are:

1. Integration of traffic assessment system and warning communication system.

2. Interconnection of the common information and communication technologies which are the internet, mobile technology and database.

3. Division of tasks to modules and enabling encapsulation and abstraction.

4. Development of interfaces between the modules of the proposed system and between the system and mobile network.

5. Understanding the levels of severity of traffic snarls and accordingly assigning priority of communication.

6. Automated initiation, processing and output of information by the proposed system.

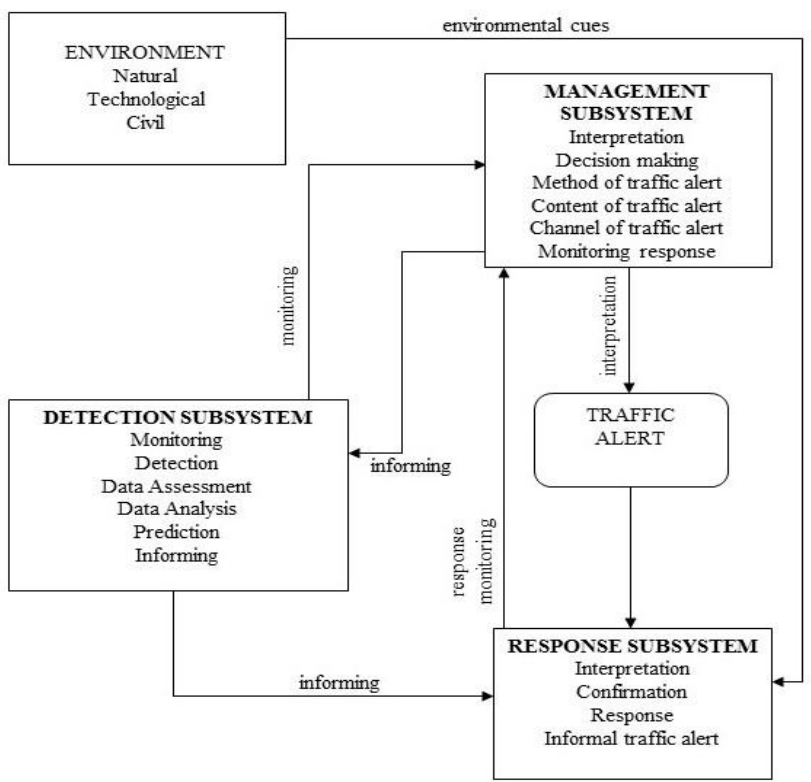

Figure 1. The general components of a geographical information integrated traffic alert system (Pseudocode, 2017).

\subsection{Proposed Strategy}

The present architecture overcomes the above problems by providing a system and method for gathering and sending monitored traffic data via a short messaging system message over a wireless network. The traffic situation / positioning data is already available to us as shown in Figure 2. The method includes determining the location of the mobile communicator from location data provided by mobile communication stations of a mobile communication network. The location of the mobile communicator can be extracted e.g. by a triangulation method on the data, collected from the cell phone towers. Also, the location of the mobile communicator can be extracted from data provided by global positioning system or a related cell-phone GPS system. The system includes a database having stored thereon a profile relating to a user of a mobile device (Pseudocode, 2017).

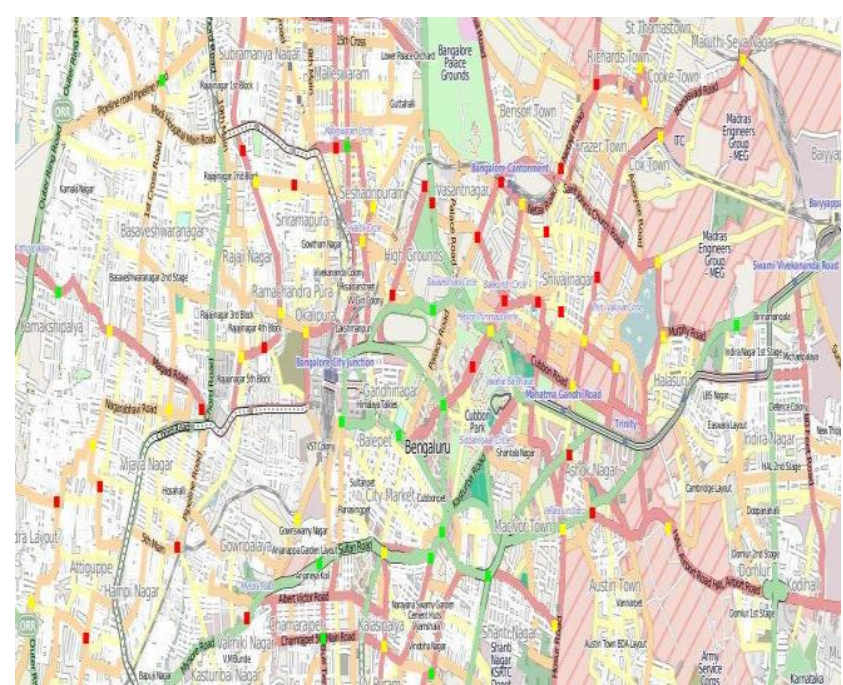

Figure 2. Input geospatial database from city of Bangalore (pixel-wise georeferenced) for the alert system, from real-time traffic on roads. Red dot - congestion, Yellow dot - piling up, Green dot - clear way.

The system also includes a server, wherein the server is configured to receive traffic information relating to a route and location information relating to the mobile device, and wherein the server is configured to send a traffic alert to the mobile device when the traffic information and the location information indicate that the mobile device is traveling toward traffic congestion (Figure 3 and 4). The method is of monitoring the traffic alert information may include monitoring information provided by one of a state transportation department, a police department, a law enforcement agency, or any mobile enterprise. If the data from the traffic database do not indicate that an traffic alert is possible, the system monitors the data for further traffic alerts.

If the data from the traffic database indicate that an traffic alert is possible, the system advances by querying the location server where the mobile device is located and/or the direction of travel of the mobile device. Based on the position and/or the direction of travel of the mobile device the system determines whether a traffic alert is needed (Figure 3,4). For example, if the mobile device is located in proximity to a traffic accident that is causing congestion and/or if the mobile device is traveling in the direction of the traffic congestion, a traffic alert to the mobile device is appropriate. Also, the system determines whether the mobile device is within a predetermined distance from the source of the traffic congestion. 
Or, the predetermined distance is specified by the subscriber of the user in the profile that is stored in the database, while exercising the option of registering. So the idea is to develop a system which can fulfill the role of a traffic alert system, comprising a database having stored thereon a profile relating to a user of a mobile device; and a server, wherein the server is configured to receive traffic information relating to a route and location information relating to the mobile device, and wherein the server is configured to send a traffic alert to the mobile device when the traffic information and the location information indicate that the mobile device is traveling toward traffic congestion.

There is a standard Universal Serial Bus (USB) for serial connection between the modem and the PC, which is also applicable for the GSM device (GSM modem). First mobile devices were connected to the computers via cable (a real serial connection). Nowadays only pluggable GSM modems use real serial port to communicate with the computer, whereas ordinary mobile phones use serial connection (they use one of the Bluetooth services virtual serial connection).

\subsection{Interfacing With The GSM Network}

The interfacing with the cellular network requires internal processing comprising the database and web servers. This maintains the actual data flow (Figure 4, 5, 6) controlled by the http/s and TCP/IP commands. After creating the data packet a http request is generated by the system. The requests are processed by the server hosting web-content module and the access to the database is provided through a TCP/IP channel. The function calls are made in sequential manner as further internal processing calls up the trigger module and communication module. Once the server command ComX present in attention (AT) command-set is executed by the communication module the connection to the modem over a physical channel RS232 is established. The terminal protocol AT (attention/initiate) communication protocol command-set directly issues modem commands and activates the modem. The AT communication protocol command-set enables dispatch of SMS by executing specific commands.

The SMS protocol program is called after the database table is accessed and mobile numbers in the region are extracted from the table. The mobile numbers are entered as command line parameters in the program thereafter the respective traffic situation messages are sent. The number of mobile numbers selected per region is fed in a loop. The SMS program is called for each number for sending SMS. It connects to the SMS gateway through the internet and this gateway forwards the traffic message to the mobile numbers (Figure 7).

And successively the GSM_Method is executed to enable interfacing between the communication module and external GSM environment. It transmits each alert message from the communication module over mobile communication network. The modem functionality is handled by the telecommunication commands from the communication module. Thus the interfacing and communication with the mobiles gets established. The executable program creates empty form-fields which have to be filled by the text strings from the traffic message. The GSM method sends the strings to be inserted into empty form-fields. At the beginning of the GSM program is the declaration of the variables to be used in a GSM environment. Next the opening of a message is handled by the program. Within the body of the program various headers are defined. For handling the body of the traffic message the program has a separate section of code. This code segment defines and implements the appropriate size limits, other SMS technical specifications and the time to communicate parameters are filled in.

The communication module (Fig. 7) is equipped with two ways of interfacing with the GSM network to send SMS messages from the warning system to mobile phones. The two methods are:

1. Connectivity of the traffic alert system to the SMS center (SMSC) or SMS gateway of a wireless carrier or SMS service provider through the internet. Subsequently the communication module sends SMS messages using a protocol / interface supported by the SMSC or SMS gateway. This is the software method of message sending.

2. Connectivity of GSM modem to the traffic alert system and execution of AT commands to instruct the GSM modem to send SMS messages. This is the hardware method.

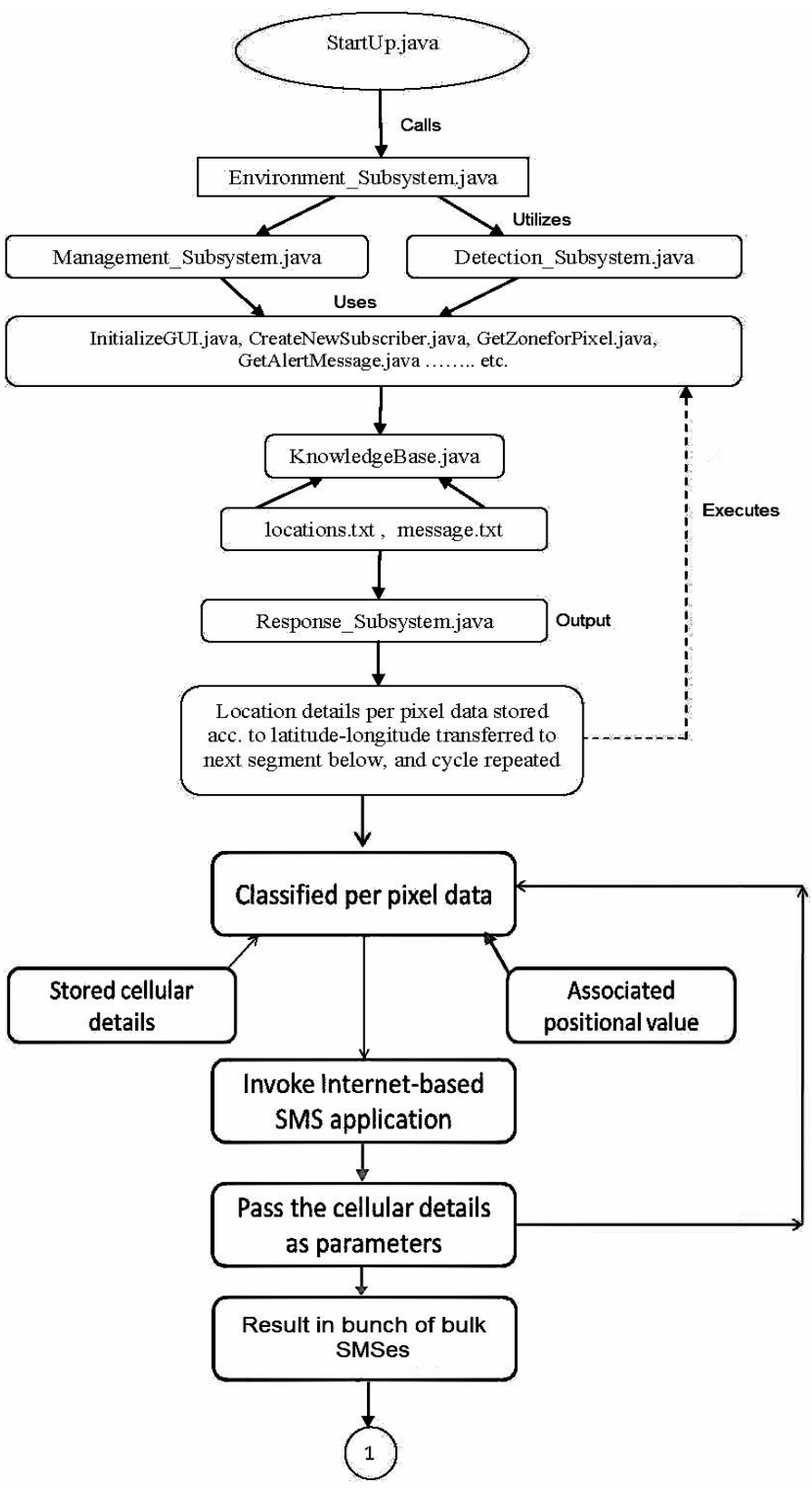

Figure 3. The flowchart of traffic alert system, top level design (Pseudocode, 2017). 
The wireless data is sent / receive from the Mobile Phone to the GSM gateway in the form of a 'struct.' The 'struct' data is passed by the GSM gateway to the Ethernet gateway using I2C. The Ethernet gateway takes each individual piece of data from the structure and post it to the MQTT broker. The MQTT broker (Mosquitto) on the Raspberry Pi picks up the data and post it via programs and operating system (Linux) sitting on the same Raspberry Pi.

The two Arduinos of the gateway are connected by 2 wires (I2C) connection. The GSM Gateway Arduino has the GSM module mounted on it while the Ethernet Gateway has Ethernet shield with the Ethernet cable connected to router (Fig. 8). The traditional GSM module uses RS232 $(2 \mathrm{v}-8 \mathrm{~V})$ with flow control (RX, TX, CTS, RTS, CTS, DTR, DSR, DCD, RI) while the newer one has direct pin out that can be hooked up with microcontroller board like Arduino.

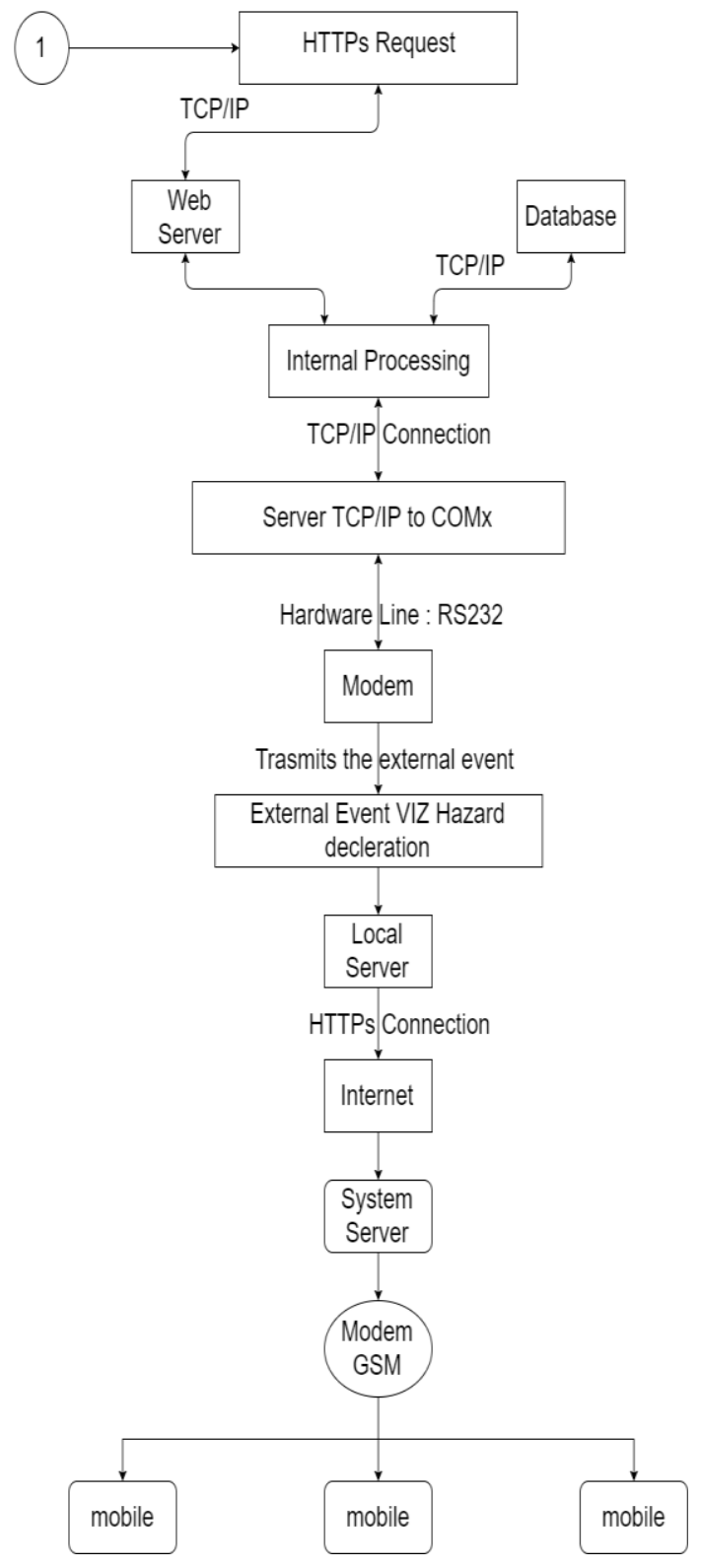

Figure 4. Continuation of traffic alert system design, bottom level flowchart (Pseudocode, 2017).

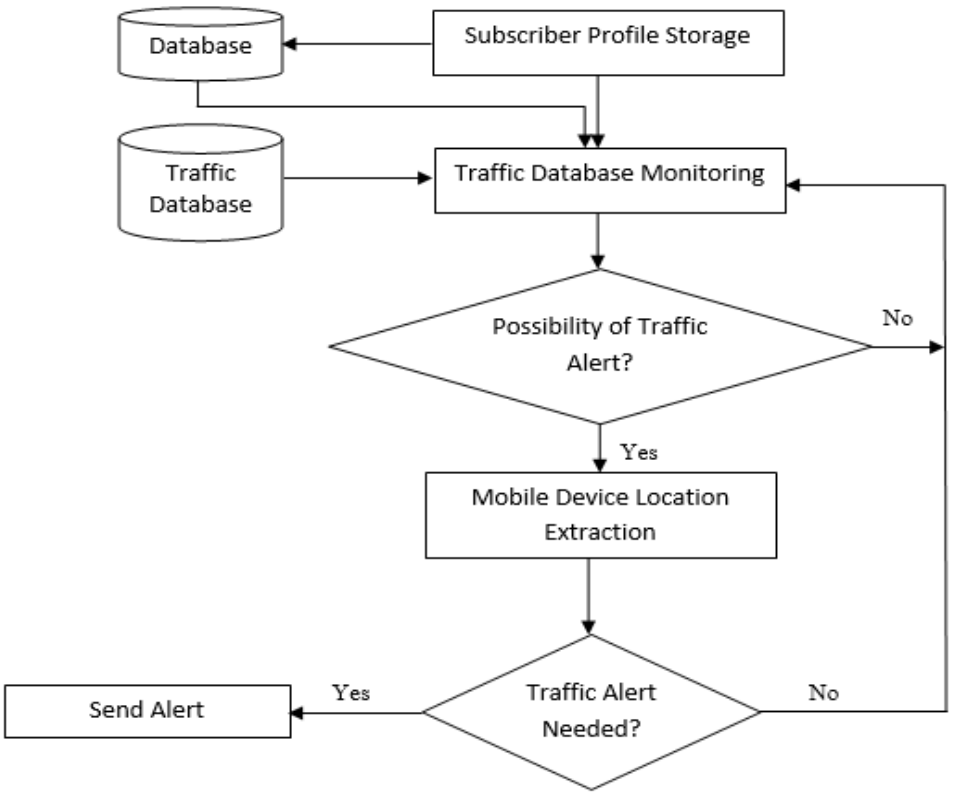

Figure 5. Traffic alert system architecture with fuctionalities modularized.

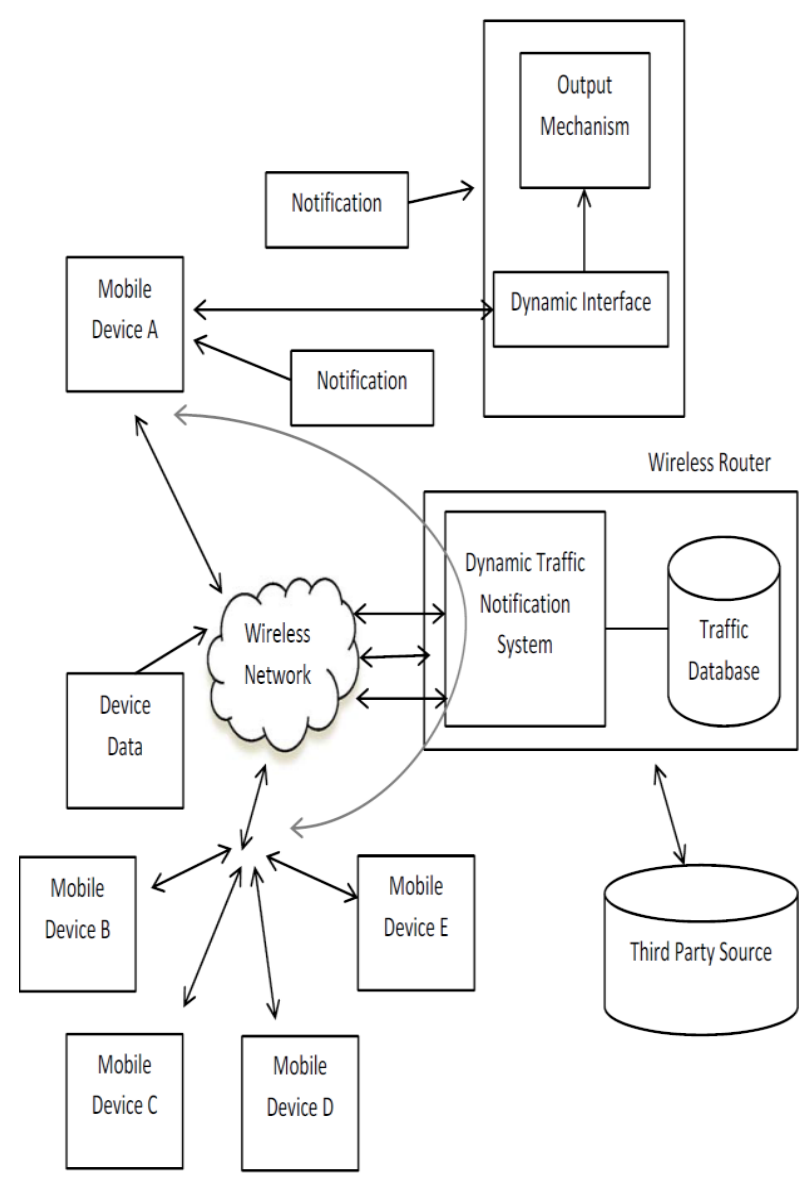

Figure 6: Integrated interfaces of modules for traffic database to mobile communication. 


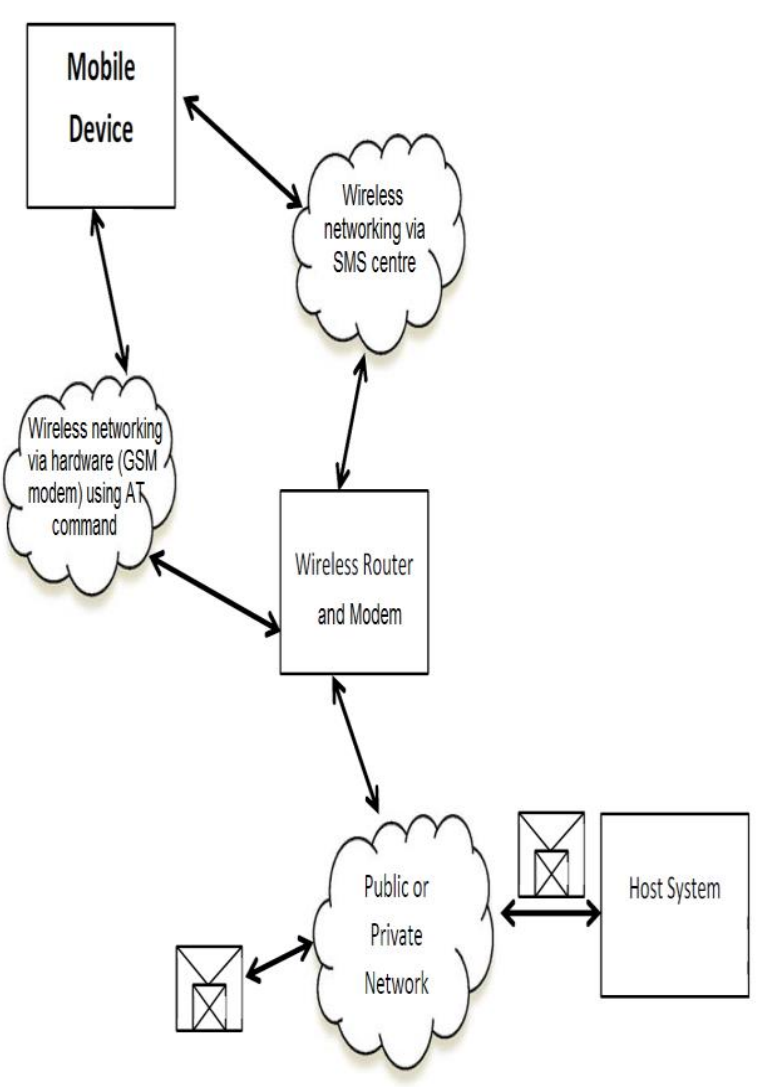

Figure 7. Internet to mobile communication through SMS.

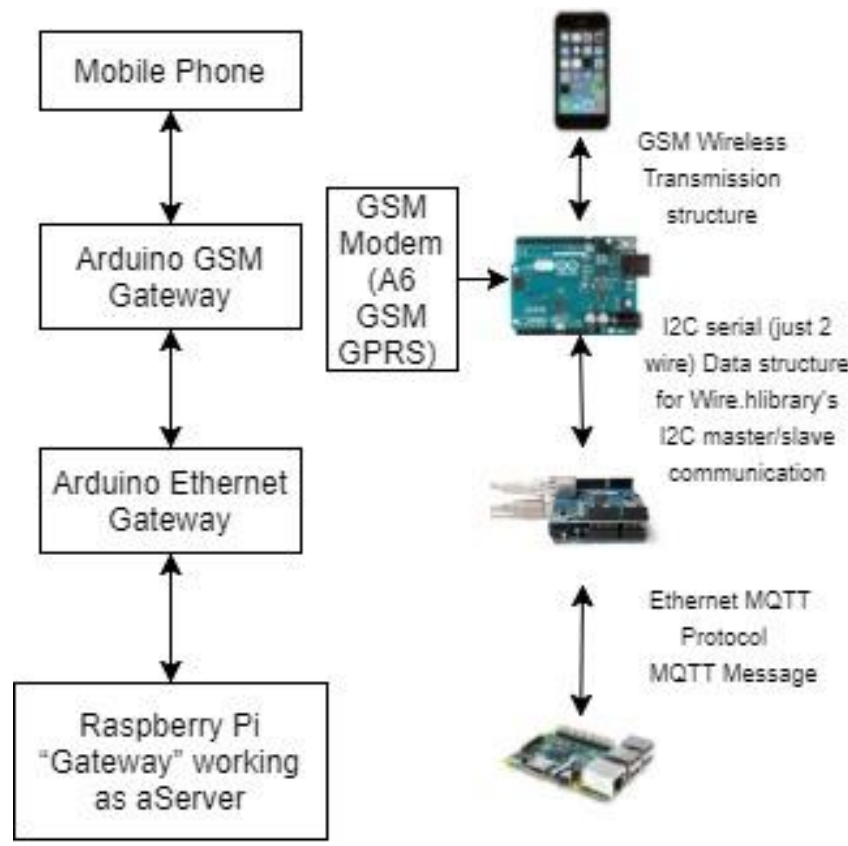

Figure 8 (a) Data flow diagram via different hardware.

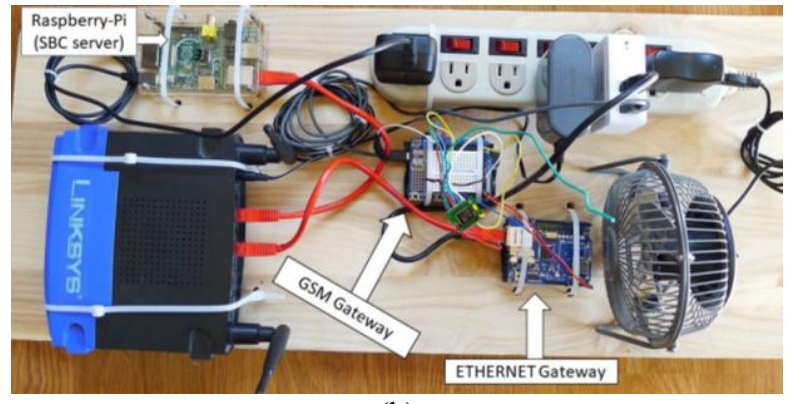

(b)

Figure 8 (b). Hardware implimentation of server.

\section{RESULTS}

The proposed system is in its developmental stage with real time traffic geospatial data sets as input. The proposed architecture design integration has been tested for warning message delivery, directions delivery and instructions delivery to users in multifarious other GIS domains within acceptable time delays where maximum time delay was 50 seconds, minimum 5 seconds and overall average 27.5 seconds. The creation and collection of a city specific traffic geospatial dataset is underway which would then be fed to the existing system as any other geospatial data. The substantiation process comprised of creating a database of latitudes, longitudes listed traffic junctions and pre-registered mobile numbers in data tables (shown in Table 1). The data tables store the regions in latitudes and longitudes for testing the system, and the mobile numbers to be communicated are residing within the latitudinal and longitudinal expanse of a predefined site in a city and its associated dynamic traffic conditions and notable tourist spots of attraction in the vicinity. The data packet is shown in Fig. 9. The table entries were selected sequentially and uninterruptedly and then the messages were transmitted 14 times 20 short messages (SMS) in a bulk to zonally identified mobile numbers, ensuing in a total number of 280. It was tabulated that 196 messages delivered within 10 seconds, rest within 40 seconds (Table 2). All generated messages were delivered and the delivery time limit was found acceptable for quick response purposes.

\begin{tabular}{|r|c|c|}
\hline client_geo_location & client_mobile & traffic_guidance_string \\
\hline $\begin{array}{c}30^{\circ} 04^{\prime} 00^{\circ} \mathrm{N} \text { and } \\
30^{\circ} 19^{\prime} 00^{\prime \prime} \mathrm{N} \\
77^{\circ} 50^{\circ} 00^{\prime \prime} \mathrm{E} \text { and } \\
77^{\circ} 65^{\prime} 00^{\prime \prime} \mathrm{E}\end{array}$ & 9444317343 & $\begin{array}{c}\text { Next right turn at signal, } \\
\text { avoid service lane }\end{array}$ \\
\hline $\begin{array}{c}30^{\circ} 20^{\prime} 00^{\prime} \mathrm{N} \text { and } \\
30^{\circ} 35^{\prime} 00^{\prime \prime} \mathrm{N} \\
77^{\circ} 66^{\prime} 00^{\prime \prime} \mathrm{E} \text { and } \\
77^{\circ} 81^{\prime} 00^{\prime \prime} \mathrm{E}\end{array}$ & 9986572110 & $\begin{array}{c}\text { 2 signals heavy jam, } \\
\text { divert towards the } \\
\text { flyover at next left turm }\end{array}$ \\
& & \\
so on ..... & 50 on .... & so on .... \\
\hline
\end{tabular}

Table 1. Example table entries for travelling subscribers' bunch data and the traffic \& travel information to be conveyed to them

\begin{tabular}{|c|c|c|c|}
\hline \multicolumn{3}{c}{ End-Marker } \\
\hline $\begin{array}{c}\text { subscriber } \\
\text { cell } \\
\text { number }\end{array}$ & city specific geo- & $\begin{array}{c}\text { Traffic message, } \\
\text { important venues } \\
\text { directions, } \\
\text { miscellaneous } \\
\text { instructions }\end{array}$ \\
\hline
\end{tabular}

Figure 9. The data packet formed from Table 1 for conveying information to traveller subscribers, also to classify type of traveller whether tourist or regular city dweller. 


\begin{tabular}{|c|c|c|c|}
\hline $\begin{array}{l}\text { Trial on } \\
\text { subscribers } \\
\text { bunch }\end{array}$ & $\begin{array}{l}\text { Min duration } \\
\text { (seconds) }\end{array}$ & $\begin{array}{l}\text { Max duration } \\
\text { (seconds) }\end{array}$ & $\begin{array}{l}\text { Average } \\
\text { delay } \\
\text { (seconds) }\end{array}$ \\
\hline $\mathbf{1}$ & $\mathbf{1 0}$ & $\mathbf{2 0}$ & $\mathbf{1 5}$ \\
\hline 2 & 13 & 45 & 29 \\
\hline 3 & 5 & 35 & 20 \\
\hline 4 & 11 & 49 & 30 \\
\hline 5 & 13 & 44 & 29 \\
\hline 6 & 12 & 46 & 29 \\
\hline 7 & 15 & 44 & 29.5 \\
\hline 8 & 7 & 45 & 19 \\
\hline 9 & 10 & 50 & 30 \\
\hline 10 & 9 & 43 & 17 \\
\hline 11 & 12 & 46 & 29 \\
\hline 12 & 11 & 41 & 29 \\
\hline 13 & 13 & 44 & 25 \\
\hline 14 & 8 & 42 & \\
\hline
\end{tabular}

Table 2. Time statistics for validating integration of system with the traffic \& travel information SMS conveyed to travellers.

\section{DISCUSSIONS}

The system addresses the issues of travellers moving into a new location where they are likely to miss places of attraction anywhere around them or likely take lengthier choices to reach otherwise shorter points of interest. Based on their situated location, travellers can be directed to neighbourhood touristic spots in a locality with various combinations of origin destination routes. This eliminates possibilities like tourists regretting missing to visit a spot of interest due to lack of location information. Since in India routes are complex and locations could be confusing to reach by paper maps, a feeder system is proposed for pin-pointing locations of significance. According to the position of the service requesting mobile, the places of interest are notified. This service could be registered into through an app or website. Currently the trial runs have been done for known mobiles identifications having simulated conditions of location details.

The system architecture is novel in terms of utilizing hybridization of open-source technologies to offer a complete end-to-end solution. The architecture facilitates compartmentalized development and upgradation. Also one aspect of novelty is the use of locations indexes for databases to keep important notifications about tourist points ready to be searched and sent. This approach is suitable for the current setting since an SMS based approach is appropriate in the Bangalore context as there is low availability of smart phone devices, and mobile phone bandwidth is low.

\section{CONCLUSIONS AND FURTHER SCOPE}

The proposed architecture has made it possible to research towards the development of an integrated traffic situation assessment and alert communication system. Integration of traffic situation assessment and warning communication has been done. Geo-spatial referencing of location, disturbances or zones of interest and communication has been achieved using automated data creation, transformation and dissemination over different interfaces. The aim being modularizing the functionalities of different technologies to form a single system integration. Achieved system functionalities abstraction and encapsulation for low maintenance and easy upgradation in future. Open-source technologies utilized for the system architecture, design and development.

A never before architecture has been introduced for integrating WebGIS with telecommunication technology. Modularization of different technologies towards development of an alert communication system leading to automated data creation, transformation and dissemination over different interfaces. Existing technologies interfaced in a novel architectural design to address a neglected domain in a way never done before - through dynamically updatable WebGIS based warning communication. The architecture of the developed alert system has been functionally automated as well as generalized enough that can be used for any traffic alert. Setup requirement has been kept to a minimum. This work publishes new architecture and novelty in addressing hazard warning techniques in sustainable way and user friendly manner. Future work is progressing to include voice messages since to read a SMS attention is diverted creating problems for the driver of a vehicle.

Hence the system can be summarized as the development of internet-SMS based automated traffic alert system. A novel architecture has been introduced where major technologies in ICT have been interfaced to give a unique ICTWebGIS based dynamic real time alert communication system. Integrating an alert system with a situation evaluation system this architecture interfaces different open-source technologies towards design and development of a warning system. The development towards complete data collection for a city e.g. Bangalore and converting to a geospatial platform is underway.

\section{ACKNOWLEDGEMENTS}

D. Bhattacharya has been funded by the European Commission through the GEO-C project (H2020-MSCA-ITN-2014, Grant Agreement number 642332, http://www.geo-c.eu/). The authors are thankful to anonymous reviewers for their constructive comments that helped improve the paper.

\section{REFERENCES}

Ajitha, T., Vanajakshi, L., Subramanian, S. C., 2013. "RealTime Traffic Density Estimation without Reliable Side Road Data." J. Comput. Civ. Eng., Permalink: http://dx.doi.org/10.1061/(ASCE)CP.1943-5487.0000310.

Barone, R.E., Giuffrè, T., Siniscalchi, S.M., Morgano, M.A., Tesoriere, G., 2014. "Architecture for parking management in smart cities", IET Intelligent Transport Systems, 8(5), pp. $445-$ 452, DOI: 10.1049/iet-its.2013.0045, Print ISSN 1751-956X, Online ISSN 1751-9578.

Bhattacharya, D., Ghosh, J. K., Boccardo, P., Samadhiya, N. K., 2011. "Wireless Hazard Communication System", Journal of Systems and Information Technology, Emerald Publishing (United Kingdom), 13(4), pp. 408-424.

Bhattacharya, D., Ghosh, J. K., Boccardo, P., Komarkova, J., 2013. "Automated Geo-Spatial System for Generalized Assessment of Socio-Economic Vulnerability due to Landslide in a Region”, European Journal of Remote Sensing (EuJRS), 46, $\quad$ pp. $\quad 379 \quad-\quad 39$, http://www.tandfonline.com/doi/abs/10.5721/EuJRS20134622 
Bhattacharya, D., Ghosh J. K., Komarkova, J., Banerjee, S., Kutoglu H. S., 2014. "Distributed GIS for Automated Natural Hazard Zonation Mapping Internet-SMS Warning towards Sustainable Society", Cogent Engineering, Taylor \& Francis Online DOI Link

Cervantes, O., Gutiérrez, E., Gutiérrez, F., Sánchez, J. A., 2016. "Social Metrics Applied To Smart Tourism", ISPRS Ann. Photogramm. Remote Sens. Spatial Inf. Sci., IV-4/W1, 117-124, https://doi.org/10.5194/isprs-annals-IV-4-W1-117-2016.

Jog, G.M., Li, S., Gerber, B.B., Brilakis, I. 2011. "Civil and Environmental Engineering Challenges for Data Sensing and Analysis", ASCE Jrnl. Computing in Civil Engineering, pp. 110-117. Permalink: http://dx.doi.org/10.1061/41182(416)14.

Korhonen, T., Väärämäki, T., Riihimäki, V., Salminen, R., Karila, A., 2012. "Selecting telecommunications technologies for intelligent transport system services in Helsinki Municipality", IET Intelligent Transport Systems, 6(1), pp. 1828, http://digital-library.theiet.org/content/journals/10.1049/ietits.2010.0090.

Ma, J., Smith, B. L., Zhou, X., 2015. "Personalized real-time traffic information provision: Agent-based optimization model and solution framework." Transportation Research Part C: Emerging Technologies,

http://dx.doi.org/10.1016/j.trc.2015.03.004.

Manasseh, C., Sengupta, R., 2010. "Middleware to enhance mobile communications for road safety and traffic mobility applications", IET Intelligent Transport Systems, 4(1), pp. $24-$ 36, DOI: 10.1049/iet-its.2009.0019 , Print ISSN 1751-956X, Online ISSN 1751-9578.

Manolis, D., Papamichail, I., Kosmatopoulos, E. B., Papageorgiou, M., 2016. "Automated tuning of ITS management and control systems: Results from real-life experiments." Transportation Research Part C: Emerging Technologies, 66, pp. 119-135, ISSN 0968-090X, http://dx.doi.org/10.1016/j.trc.2015.08.020.

Pascale, M., Nicoli, F., Deflorio, B., Chiara, D., Spagnolini U., 2012. "Wireless sensor networks for traffic management and road safety", IET Intelligent Transport Systems, 6(1), pp. 67 77.

Piao, J., McDonald, M., Hounsell, N., 2012. "Cooperative vehicle-infrastructure systems for improving driver information services: an analysis of COOPERS test results", IET Intelligent Transport Systems, 6(1), pp. 9-17, http://digitallibrary.theiet.org/content/journals/10.1049/iet-its.2010.0169.

Pseudocode, 2017. "Schema for Tourist Guidance API", https://github.com/Friscon/TouristGuidanceAPI

Semertzidis, T., Dimitropoulos, K., Koutsia, A., Grammalidis, N., 2010. "Video sensor network for real-time traffic monitoring and surveillance", IET Intelligent Transport Systems, 4(2), pp. 103 - 112, DOI: 10.1049/iet-its.2008.0092, Print ISSN 1751-956X, Online ISSN 1751-9578.

Sisiopiku, V.P., Elliott, J.R., 2005. "Active Warning Systems: Synthesis." J. Transp. Eng., 131(3), pp. 205-210, Permalink: http://dx.doi.org/10.1061/(ASCE)0733-947X(2005)131:3(205).
Sun, J., Liu, H. X., 2015. "Stochastic eco-routing in a signalized traffic network." Transportation Research Part C: Emerging Technologies, 59, pp. 32-47.

Wang, Z., Zlatanova, S., Steenbruggen, J., 2016. "Traffic Incident Management In The Presence Of Hazards", ISPRS Ann. Photogramm. Remote Sens. Spatial Inf. Sci., IV-4/W1, 45-49, https://doi.org/10.5194/isprs-annals-IV-4-W1-45-2016.

Yuan, Q., Liu, Z., Li, J., Zhang, J., Yang, F., 2014. "A traffic congestion detection and information dissemination scheme for urban expressways using vehicular networks." Transportation Research Part C: Emerging Technologies, 47, pp. 114-127.

Zarif, J. E., Hobeika, A., Rakha, H., 2003. "Evaluating a Detection and Warning System to Deter No-Passing Zone Violations", ASCE Journal of Transportation Engineering, 129(6), pp. 590-599, DOI link http://ascelibrary.org/doi/abs/10.1061/\%28ASCE\%290733$947 \mathrm{X} \% 282003 \% 29129 \% 3 \mathrm{~A} 6 \% 28590 \% 29$.

Zhao, J., Zhang, Y., Pan, Y., He, J., 2012. "Study on the Early Warning System of Road Passenger Transport", CICTP 2012 , pp. 1065-1076, Permalink: http://dx.doi.org/10.1061/9780784412442.109;http://ascelibrary .org/doi/abs/10.1061/9780784412442.109. 